\title{
Performance Analysis of InterpolatedShrink Method in Image De-Noising
}

\author{
J S Bhat \\ Dept. of Physics \\ Karnatak University \\ Dharwad, India-580003
}

\author{
B N Jagadale \\ Dept. Of electronics \\ Kuvempu University \\ Shankaragatta , India-577451
}

\begin{abstract}
The de-noising of an image corrupted by Gaussian noise is a classical problem in signal or image processing. An image is often corrupted by noise during its acquisition and transmission. Image de-noising is used to reduce the noise while retaining the important features in the image. Always there exists a tradeoff between the removed noise and the blurring in the image. The use of wavelet transform for signal de-noising has emerged as an important technique during the last decade. The wavelet transform is preferred over conventional Fast Fourier Transform(FFT) based image denoising technique ,because of its capability to give detailed spatial-frequency information. In this paper, we tried to analyze the performance of InterpolatedShrink method in image de-noising using various wavelet family, such as Haar,Doubechies,Symlet and Coiflets, for Gaussian noise.
\end{abstract}

\section{General Terms}

Computer Science- Image Processing.

\section{Keywords}

De-noising, Thresholding, Discrete Wavelet Transform, Gaussian noise, IntepolatedShrink

\section{INTRODUCTION}

Image de-noising is a problem of prime importance in image processing field, ranging from medical imaging to satellite imaging. With rapid growth in digital technology, the Engineers and scientists are gathering data and analyzing it at an ever increasing pace. Generally the data collected by sensors is corrupted by noise, either as a result of data acquisition process or due to interfering natural phenomena or it may be due to transmission errors. Before analyzing the image or data, the noise must be removed. In image denoising, the aim is to suppress noise as much as possible while retaining the important image features. Image de-noising still remains a challenge for researchers since a tradeoff between the removed noise and the blurring in the image always exists.

In recent years, lot of research has been done on image denoising[1-3], Basically there are two approaches to image denoising, spatial filtering method and transform domain filtering method [4]. A conventional way to remove noise from a noisy image is to use the spatial filter. Spatial filters are also classified into linear and non-linear filters. The spatial filters usually smooth the image to reduce the noise, but, in the process also blur the image. To overcome the weakness of the spatial filtering, several new techniques have been developed that improve on spatial filters by removing the noise more effectively while preserving the image features. A different class of techniques exploit the decomposition of image into the wavelet basis and de-noise the image by shrinking the wavelet coefficients[5-7].

This paper is organized as follows. Section II, gives brief review of image de-noising method by Interpolatedshrink.
The Experimental results and discussions are presented in section III and finally, we give conclusion in Section IV.

\section{REVIEW OF IMAGE DENOISING METHOD BY INTERPOLATED SHRINK}

Wavelet based image de-noising methods have been very effective because of their ability to capture the energy of a signal in few energy transform values. The multi-resolution analysis performed by the wavelet transform is one of the powerful tools. In the wavelet domain, thresholding is used to separate the information from noise. Donoho and Johnstone $[8,9]$ have developed several wavelet de-noising methods by thresholding the wavelet coefficients arising from the standard discrete wavelet transform.

An image $B(i, j)$ corrupted by Gaussian noise $Z(i, j)$ can be represented as $B(i, j)=A(i, j)+\sigma Z(i, j)$, where $Z(i, j)$ is noise free image and $\sigma$ the standard deviation of noise.

The Donoho's wavelet based de-noising scheme can be summarized as follows:

1. Transform the noisy image $\mathrm{B}(\mathrm{i}, \mathrm{j})$ into an orthogonal domain by 2D Discrete Wavelet Transform (DWT).

2. Apply soft or hard thresholding to the resulting wavelet coefficients by the threshold $\lambda=\sigma \sqrt{2 \operatorname{logn}^{2}}$.

3. Perform inverse 2D discrete wavelet transform to obtain the de-noised image.

The DWT of image is a non redundant image representation with better spatial and spectral localization of image formation, compared to other multi-scale representations such as Gaussian and Laplacian pyramid. An image can be decomposed into a sequence of different spatial resolution images using DWT. The method is also called decimated wavelet transform, since it decimates the signal into sub bands. With DWT an image can be decomposed more than once. Decomposition can be continued until the desired level is reached[10]. The Gaussian noise will nearly average out in low frequency wavelet coefficients and only detail wavelet coefficients need to be thresholded. A different class of methods exploits the decomposition of the image into wavelet basis and shrinks the wavelet coefficients to de-noise the image $[11,12]$. The shrinkage rule defines, how we apply the threshold. Here threshold plays important role in denoising process. Finding an optimum threshold is a tedious process because a small threshold value will retain the noisy coefficients, where as a large threshold value leads to the loss of coefficients that carry image signal details. There are two main approaches, hard thresholding, is the simplest method and is a keep or kill method whereas soft thresholding has nice mathematical properties and it shrinks the coefficients 
above the threshold in absolute value. It is a shrink or kill method.

The hard threshold signal

$$
\begin{array}{ccc}
X \text { is } & X & \text { if }|\mathrm{X}|>t h r \\
X \text { is } & 0 & \text { if }|\mathrm{X}|<t h r
\end{array}
$$

The soft threshold signal

$$
\begin{array}{ll}
X \text { is } \quad(|\mathrm{X}|-t h r) & \text { if }|\mathrm{X}|>t h r \\
\mathrm{X} \text { is } 0 & \text { otherwise }
\end{array}
$$

The hard thresholding deletes all coefficients that are smaller than the threshold, and keeps the others unchanged. On the other hand soft thresholding also deletes the coefficients under the threshold, but scales the ones that are left. Hard thresholding creates discontinuities in the reconstructed signal, while soft does not. The BayesShrink, VisuShrink and SureShrink along with NormalShrink[13],are well known methods based on wavelet transform. These approaches have been proposed, by considering the influence of other wavelet coefficients on the current wavelet coefficient, to be thresholded. The motivation of this idea is that a large wavelet coefficient will probably have large wavelet coefficients at its neighbors. This is because even when coefficients are uncorrelated and close, there are still significant higher order neighbor correlations, like a strong positive covariance in amplitude between neighbor coefficients. Cai and Silverman [14] proposed a method that takes the immediate neighbor coefficients into account for 1D signal. Here $d_{j, k}$ is the set of wavelet coefficients of signal corrupted with noise and if

$$
S_{j, k}^{2}=d_{j, k-1}^{2}+d^{2}{ }_{j, k}+d^{2}{ }_{j, k+1} \leq \lambda^{2},
$$

then, wavelet coefficients $d_{j, k}$ are set to zero, otherwise shrink it using

$$
d_{j, k}=d_{j, k}\left(1-\frac{\lambda^{2}}{S^{2}{ }_{j, k}}\right)
$$

where $\lambda=\sigma \sqrt{2 \log \mathrm{n}^{2}}$ and $\mathrm{n}$ is length of the signal. 2D wavelet transform is performed for image de-noising. At every decomposition level, four frequency sub bands are created. This process continues until the required level is reached. Some more methods have been proposed based on statistical modeling of wavelet coefficients [15,16]. By extending the idea of Cai and Silverman for 2D image case, Chen et al.[17] proposed new method namely NeighShrink which thresholds the wavelet coefficients according to the sum of the squares of all the wavelet coefficients within a neighborhood window.

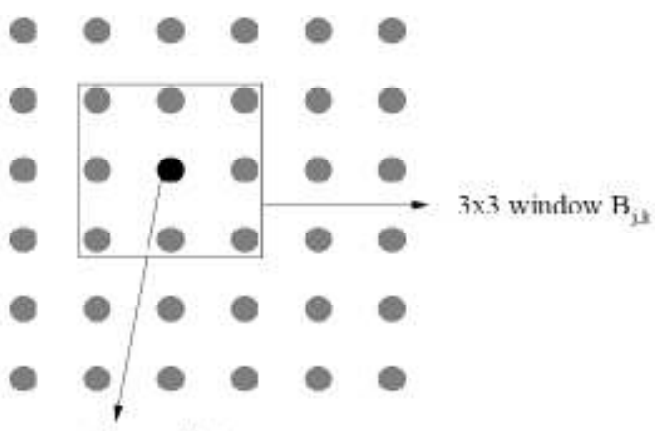

wavelet coefficient

to be thresholded

Figure 1. Neighborhood window with size $3 \times 3$.

In the new approach called InterpolatedShrink [18], for each noisy wavelet coefficient $W_{i j}$ to be shrinked, it incorporates a square neighboring window $\mathrm{B}_{\mathrm{ij}}$ centered at it. The neighboring window size can be represented as $\mathrm{LxL}$, where $\mathrm{L}$ is a positive odd number. Figure 1 , shows a $3 \times 3$. neighboring window centered at the wavelet coefficient to be shrinked. The key point is that the interpolated value $\mathrm{M}_{\mathrm{i}, \mathrm{l}}^{2}$ of the neighborhood is determined by sorting the window coefficients. The window coefficients are replaced by the new interpolated value. In this method, there is no abrupt change in the neighboring window coefficients around the current wavelet coefficients, which in turn help in determining the threshold value. Also, even if there is an abrupt change at the edges, the new value will help in preserving the edge information. The wavelet coefficients are then thresholded, according to sum of squares of all the wavelet coefficients $S^{2}{ }_{j, k}$ within a neighborhood window. The interpolated median value is slightly greater than median value of the sorted window coefficients, which is more appropriate in determining the threshold value and preservation of edge information. The sum is calculated using the new coefficients in the formula

$$
S_{j, k}^{2}=\sum_{(i, l) \epsilon B_{j, k}} M_{i, l}^{2}
$$

and different wavelet coefficient sub bands are thresholded. Then Shrink it according to the following formula:

$$
d_{j, k}=d_{j, k} B_{j, k}
$$

with $\mathrm{B}_{\mathrm{j}, \mathrm{k}}=\left(1-\frac{\lambda^{2}}{\mathrm{~S}^{2} \mathrm{j}, \mathrm{k}}\right)+$. The + sign in the formula means it takes only positive value, and $\lambda=\sigma \sqrt{2 \operatorname{logn}^{2}}$ is the threshold value. 


\section{RESULTS AND DISCUSSIONS}

In this section, performance of InterpolatedShrink method in image de-noising is analyzed experimentally using various wavelet family, such as Haar, Doubechies, Symlet and Coiflets. The experiments are conducted on some natural grayscale test images like Lena and Barbera of size $512 * 512$ at different Gaussian noise levels, Standard deviation $\sigma$ is varied from 5 to 50 in steps of 5 and results are tabulated. In Table 1, we present consolidated results of de-noising values for Lena and Barbara Images. Figure 2 and 3, shows some denoised image results for lena and Barbara with Gaussion noise $(\sigma=25$ ). The peak signal-to-noise ratio (PSNR) in decibels $(\mathrm{dB})$, is defined as as

$P S N R=10 \times \log \frac{255^{2}}{M S E}(d B)$ with

$M S E=\frac{1}{m n} \sum_{i=0}^{m-1} \sum_{j=0}^{n-1}(I(i, j)-K(i, j))^{2}$

where I and $\mathrm{K}$ being the original image and de-noised image, respectively.

Also from the Table 1, it is observed that for the Lena image, The wavelet $\mathrm{Db} 6$ gives better performance at low noise levels (for $\sigma=5,10$ and 15), where as $\mathrm{Db} 2$ gives better results for higher noise level (for $\sigma=20,25,30,35,45$ and 50) . In case of Barbara image, Coif4 gives the best results at low noise levels(for $\sigma=5,10,15$ and 20) and Db6 gives intermediate noise levels (for $\sigma=25,30,35$ and40) and $\mathrm{Db} 2$ at low noise levels(for $\sigma=45$ and 50).

Table 1. Image de-noising results for different levels of Gaussian noise added images using various wavelet filters

\begin{tabular}{|l|l|l|l|l|l|l|l|l|}
\hline $\begin{array}{l}\text { Noise std. } \\
\text { deviation }\end{array}$ & \multicolumn{9}{|c|}{ Lena Image } \\
\hline$\sigma$ & Haar & Db2 & Db4 & Db6 & Db8 & Db10 & Sym2 & Sym4 \\
\hline$\sigma=05$ & 36.5359 & 36.6788 & 37.7811 & $\mathbf{3 7 . 8 2 8 7}$ & 37.3746 & 37.3736 & 36.6788 & 37.1246 \\
\hline$\sigma=10$ & 32.1259 & 32.0735 & 34.3561 & $\mathbf{3 4 . 4 2 2 2}$ & 33.5091 & 33.0330 & 32.0735 & 33.0080 \\
\hline$\sigma=15$ & 29.4913 & 29.3892 & 32.3257 & $\mathbf{3 2 . 3 6 9 9}$ & 31.0748 & 30.3248 & 29.3892 & 30.5676 \\
\hline$\sigma=20$ & 27.5953 & 27.4839 & $\mathbf{3 0 . 8 6 2 1}$ & 30.8301 & 29.2590 & 28.3059 & 27.4839 & 28.6982 \\
\hline$\sigma=25$ & 26.1078 & 25.8766 & $\mathbf{2 9 . 6 9 6 8}$ & 29.6348 & 27.7989 & 26.6499 & 25.8766 & 27.2006 \\
\hline$\sigma=30$ & 24.8748 & 24.5254 & $\mathbf{2 8 . 7 2 7 2}$ & 28.6404 & 26.5736 & 25.2853 & 24.5354 & 25.8813 \\
\hline$\sigma=35$ & 23.8094 & 23.3830 & $\mathbf{2 7 . 9 0 2 3}$ & 27.7845 & 25.5232 & 24.1099 & 23.3830 & 24.7898 \\
\hline$\sigma=40$ & 22.8745 & 23.7200 & $\mathbf{2 7 . 1 6 9 3}$ & 27.0364 & 24.5965 & 23.0804 & 23.7200 & 23.8335 \\
\hline$\sigma=45$ & 22.0354 & 22.8711 & $\mathbf{2 6 . 5 2 7 0}$ & 26.2762 & 23.7080 & 22.1642 & 22.8711 & 22.9690 \\
\hline$\sigma=50$ & 21.2765 & 21.1015 & $\mathbf{2 5 . 9 4 5 5}$ & 25.6583 & 23.0198 & 21.3716 & 22.1015 & 22.1925 \\
\hline
\end{tabular}

\begin{tabular}{|l|l|l|l|l|l|l|l|l|}
\hline $\begin{array}{l}\text { Noise std. } \\
\text { deviation }\end{array}$ & \multicolumn{9}{|c|}{ Lena Image } \\
\hline$\sigma$ & Sym6 & Sym8 & Sym10 & Coif1 & Coif2 & Coif3 & Coif4 & Coif5 \\
\hline$\sigma=05$ & 37.6852 & 37.6483 & 37.3012 & 37.4442 & 37.5556 & 37.7628 & 37.6240 & 37.6999 \\
\hline$\sigma=10$ & 34.0559 & 34.1390 & 33.08887 & 33.9001 & 33.9352 & 34.2600 & 33.9499 & 33.9707 \\
\hline$\sigma=15$ & 31.8230 & 32.0160 & 30.4080 & 31.8973 & 31.7083 & 32.1025 & 31.6687 & 31.4166 \\
\hline$\sigma=20$ & 30.1571 & 30.4020 & 28.4150 & 30.4420 & 30.0410 & 30.4928 & 29.9623 & 29.3772 \\
\hline$\sigma=25$ & 28.8037 & 29.0947 & 26.8573 & 29.2659 & 28.6987 & 29.1952 & 28.5641 & 27.6984 \\
\hline$\sigma=30$ & 27.6613 & 28.5701 & 25.5098 & 28.3052 & 27.5657 & 28.0990 & 25.7734 & 26.2632 \\
\hline$\sigma=35$ & 26.6698 & 27.0239 & 24.3661 & 27.4815 & 26.7882 & 27.1437 & 24.6264 & 24.9793 \\
\hline$\sigma=40$ & 23.7864 & 26.1708 & 23.3463 & 26.7488 & 25.9292 & 26.2994 & 23.9171 & 23.9624 \\
\hline$\sigma=45$ & 24.9960 & 25.4067 & 22.4382 & 26.0905 & 25.1750 & 25.5439 & 22.7166 & 22.8959 \\
\hline$\sigma=50$ & 24.2776 & 24.7128 & 21.6187 & 25.4948 & 24.4715 & 24.8542 & 21.9017 & 22.0895 \\
\hline
\end{tabular}




\begin{tabular}{|l|l|l|l|l|l|l|l|l|}
\hline $\begin{array}{l}\text { Noise std. } \\
\text { deviation }\end{array}$ & \multicolumn{9}{|l|}{ Barbara Image } \\
\hline$\sigma$ & Daar & Db2 & Db4 & Db6 & Db8 & Db10 & Sym2 & Sym4 \\
\hline$\sigma=05$ & 35.5937 & 35.9649 & 36.6817 & 36.7854 & 36.1820 & 36.5012 & 35.9649 & 36.0982 \\
\hline$\sigma=10$ & 30.5467 & 30.9830 & 32.4390 & 32.6656 & 31.7224 & 31.8556 & 30.9830 & 31.5748 \\
\hline$\sigma=15$ & 27.6790 & 28.7239 & 30.0730 & 30.2998 & 29.0038 & 29.7587 & 28.7239 & 28.7572 \\
\hline$\sigma=20$ & 25.7045 & 26.8738 & 28.4213 & 28.6233 & 27.1403 & 26.9776 & 26.8738 & 26.6543 \\
\hline$\sigma=25$ & 24.1695 & 25.4280 & 27.1128 & $\mathbf{2 7 . 3 6 6 0}$ & 25.7838 & 25.3910 & 25.4280 & 25.2193 \\
\hline$\sigma=30$ & 22.8989 & 24.2785 & 26.0661 & $\mathbf{2 6 . 3 4 1 1}$ & 24.5913 & 24.0627 & 24.2785 & 24.369 \\
\hline$\sigma=35$ & 21.9422 & 23.2905 & 25.4368 & $\mathbf{2 5 . 5 0 9 6}$ & 23.5770 & 22.9514 & 23.2905 & 23.0463 \\
\hline$\sigma=40$ & 21.0371 & $22-4327$ & 24.7521 & $\mathbf{2 4 . 7 5 8 2}$ & 22.7082 & 21.9592 & 22.4327 & 22.1823 \\
\hline$\sigma=45$ & 20.2179 & 21.6718 & $\mathbf{2 4 . 1 5 8 8}$ & 24.1387 & 21.9370 & 21.1028 & 21.6718 & 21.4217 \\
\hline$\sigma=50$ & 19.6066 & 20.9765 & $\mathbf{2 3 . 6 3 9 6}$ & 23.5939 & 21.2453 & 20.3064 & 20.9765 & 20.7464 \\
\hline
\end{tabular}

\begin{tabular}{|l|l|l|l|l|l|l|l|l|}
\hline $\begin{array}{l}\text { Noise std. } \\
\text { deviation }\end{array}$ & \multicolumn{9}{|c|}{ Barbara Image } \\
\hline$\sigma$ & Sym6 & Sym8 & Sym10 & Coif1 & Coif2 & Coif3 & Coif4 & Coif5 \\
\hline$\sigma=05$ & 36.7450 & 36.8413 & 36.6206 & 36.2800 & 36.7313 & $\mathbf{3 6 . 8 8 0 6}$ & 36.2196 & 36.4271 \\
\hline$\sigma=10$ & 32.1742 & 32.4702 & 31.8477 & 31.7184 & 32.3376 & $\mathbf{3 2 . 5 5 3 8}$ & 31.4448 & 31.7813 \\
\hline$\sigma=15$ & 29.7038 & 29.8850 & 28.9977 & 29.3952 & 29.8746 & $\mathbf{3 0 . 3 2 6 1}$ & 28.8384 & 29.1274 \\
\hline$\sigma=20$ & 27.9540 & 28.0388 & 26.9610 & 27.7542 & 28.1816 & $\mathbf{2 8 . 4 8 9 4}$ & 26.9954 & 27.2612 \\
\hline$\sigma=25$ & 26.6373 & 26.6071 & 25.4081 & 26.5085 & 26.8592 & 27.1015 & 25.8046 & 25.6446 \\
\hline$\sigma=30$ & 25.6059 & 25.5006 & 24.1290 & 25.8819 & 25.7209 & 26.0258 & 25.9802 & 24.4432 \\
\hline$\sigma=35$ & 24.8637 & 24.5868 & 23.0251 & 25.1284 & 24.8860 & 25.1178 & 24.8302 & 23.3843 \\
\hline$\sigma=40$ & 24.2317 & 23.8104 & 22.0747 & 24.4816 & 24.1559 & 24.3297 & 23.9774 & 22.4240 \\
\hline$\sigma=45$ & 23.3262 & 23.1420 & 21.2241 & 23.7182 & 23.4942 & 23.6365 & 23.2477 & 21.5757 \\
\hline$\sigma=50$ & 22.6672 & 22.5629 & 20.4604 & 23.1957 & 22.9007 & 23.0167 & 22.5563 & 20.7849 \\
\hline
\end{tabular}




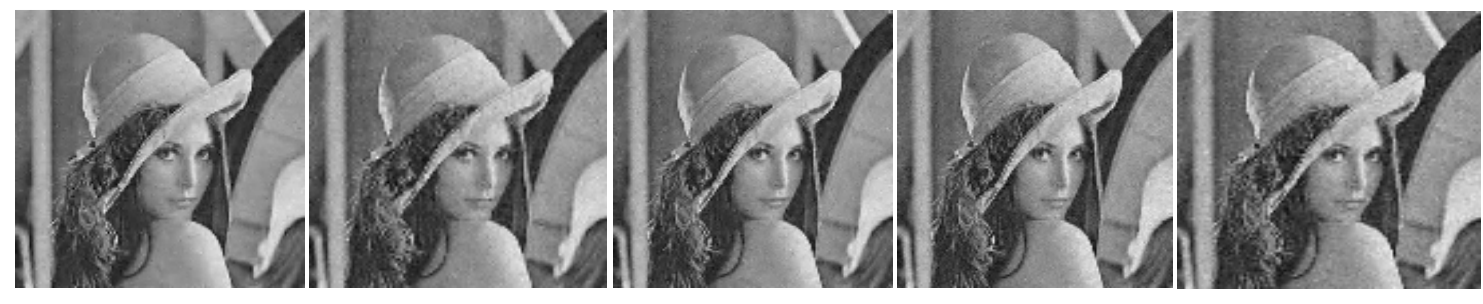

Coif1-29.2659(db) Coif2-28.6987 (db) Coif3-29.1952(db) Coif4-28.5641 db) Coif5-27.6984(db)

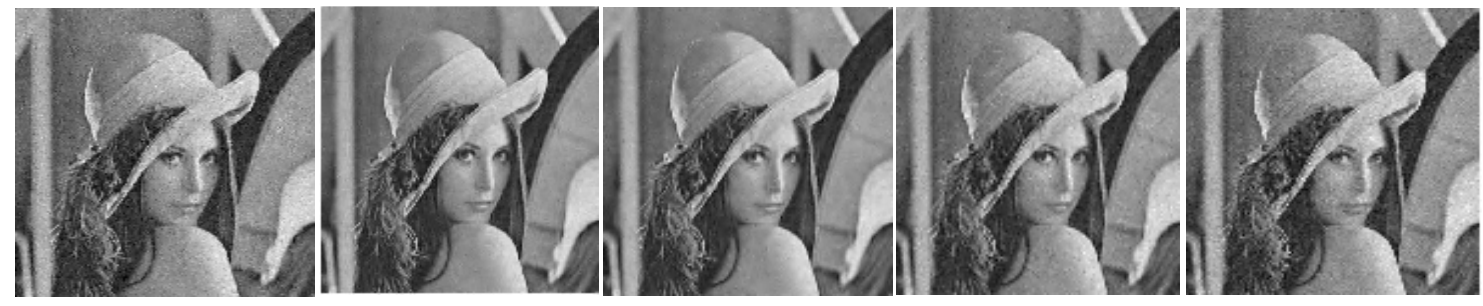

Db2- 25.8766(db) Db4-29.6968 (db) Db6-29.6348(db) Db8-27.7989 (db) Db10-26.6499(db)

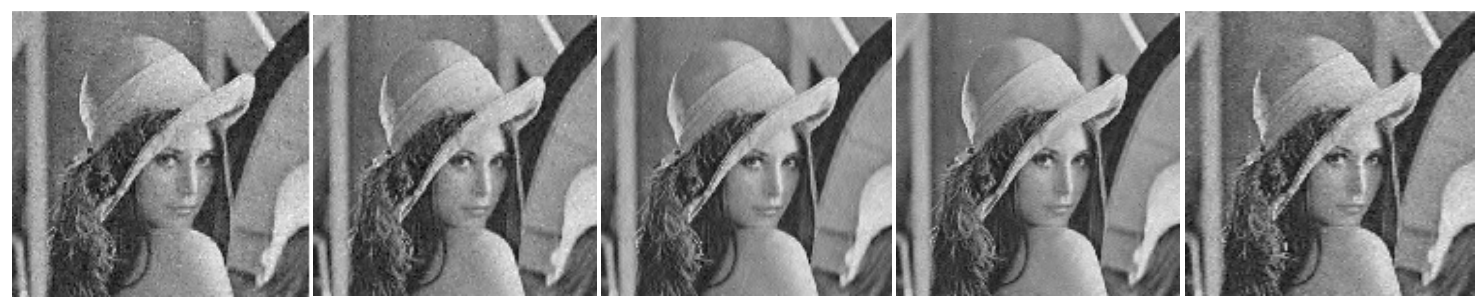

Sym2- 25.8766(db) Sym4-27.2006(db) Sym6-28.8037 (db) Sym8-29.0947(db) Sym10-26.8573(db)

Figure 2. Denoising results for Lena image with Gaussian noise $(\sigma=25$ )
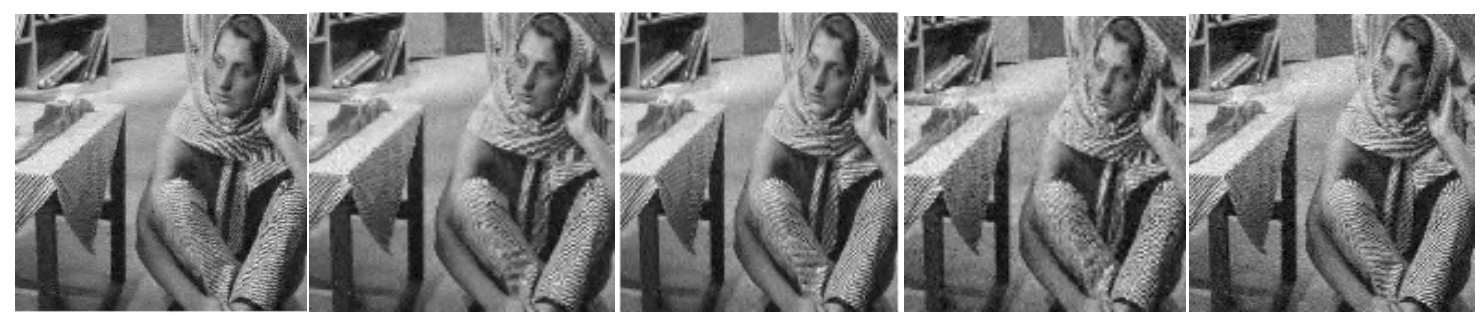

Coif1 -26.5085(db) Coif2-26.8592(db) Coif3-27.1015(db) Coif4-25.8046(db) Coif5-25.6446 (db)
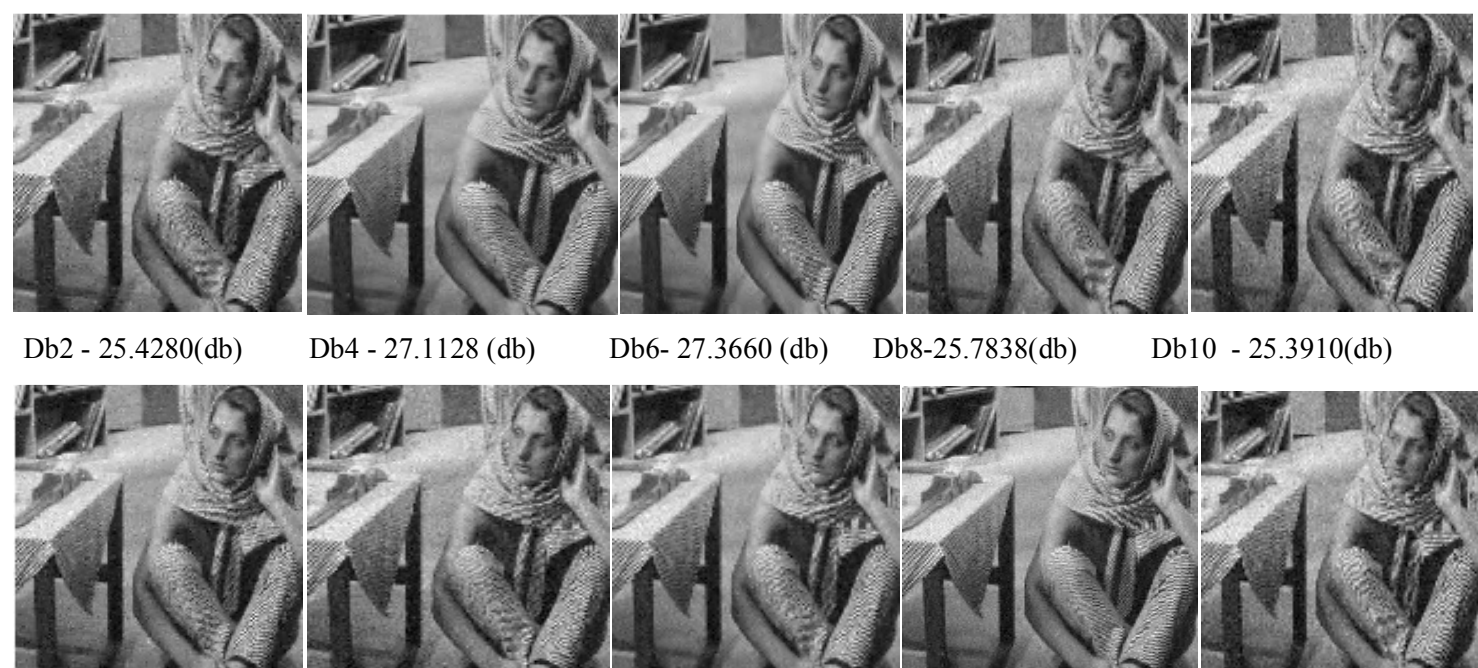

Db4 - $27.1128(\mathrm{db}) \quad$ Db6- $27.3660(\mathrm{db}) \quad$ Db8-25.7838(db)

Db10 - 25.3910(db)

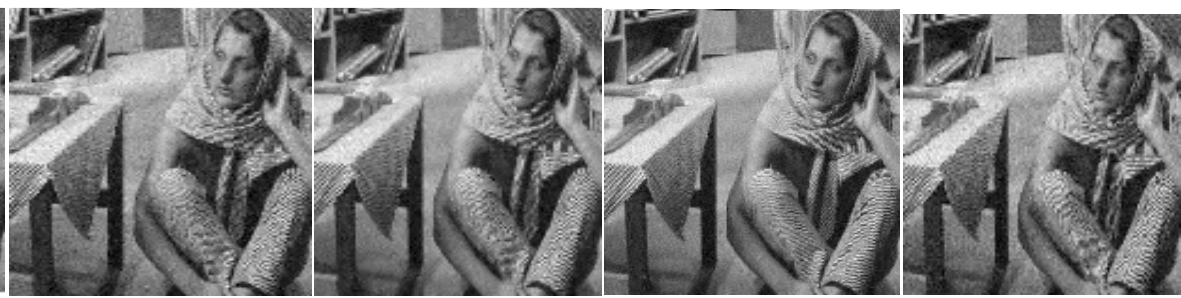

Sym2- 25.4280(db)

Sym4 - $25.2193(\mathrm{db})$

Sym6 - 26.6373(db) Sym8-26.6071 (db)

Sym10- 25.4081(db)

Fig 3. Denoising results for Barbara image with Gaussian noise ( $\sigma=25$ ) 


\section{CONCLUSION}

In This paper,The performance analysis of InterpolatedShrink method for image de-noising is evaluated. The InterpolatedShrink method is applied for de-noising images with Gaussian Noise, using various wavelet families, such as Haar, Doubechies, Symlet and Coiflets. The experimental results for different wavelets show that, the highest PSNR(dB) varies with varying noise levels. In general, InterpolatedShrink method combined with wavelet filter Db4 produces better results for removing Gaussian noise at higher levels.

\section{REFERENCES}

[1] A. K. Jain, 1989. Fundamentals of digital image processing. Prentice-Hall Inc., Englewood Cliffs, New Jersey.

[2] J. S. Lee, 1980. Digital image enhancement and noise filtering by use of local statistics. IEEE trans. On PAMI, Vol.2, pp. 165-168.

[3] A Bruce and H. Gao, Applied, 1996. Wavelet Analysis with S-PLUS. Springer Verlag.

[4] Motawani M. C., Gadiya M. C., Motwani R. C. and Harris Jr. F. C. 2004. Survey of Image Denoising Techniques. proceedings of GSPx, Santa Clara, CA.

[5] R. T. Ogden, 1997. Essential Wavelets for Statistical Applications and data Analysis. Bikhauser.

[6] B.Vidakovic, 1999. Staistical Modeling by Wavelets, Wiley Series in Probability and Statistics. Jhon Wily \& Sons, Inc.

[7] Y. Oppenheim, J. M. Poggi, M. Misiti, Y. Misiti, 2001. Wavelet Toolbox. The MathWorks, Inc., Natick, Massachusetts. (April 2001).01760

[8] D. Donoho, 1993. De-Noising by SofttThresholding. IEEE Trans. Info. Theory, vol.43, pp.933-936.
[9] D. Donoho and I. Jonstne, 1995. Adapting to unknown Smoothness via wavelet shrinkage. Journal of the American Statistical Association, vol. 90, (December 1995) pp.1200-1244,

[10] S. Mallat, 1998. A Wavelet tour of Signal processing. Academic Press, San Diego, USA.

[11] Stein, C. 1981. Estimation of the mean of a multivaarte normal distribution. Ann. Statist, vol.9, 1135-1151.

[12] S. G. Chang, B. Yu, and M. Vetterli, 2000. Adaptive wavelet thresholding for image denoising and compression. IEEE Trans. Image Processing, vol. 9, pp.1532-1546.

[13] Lakhavinder kaur, Savita Gupta and R. C. Ghauhan, 2002. Image Denoising using Wavelet Thresholding. Third conference on Computer Vision, Graphics and Image Processing, (Dec 16-18 2002). India

[14] T .T. Cai and B. W. Silverman, 2001 Incorporating information on neighboring coefficients into wavelet estimation. Sankhya A, vol. 63, pp. 127-148.

[15] M. K. Mihcak, K. R. I Kozintsev and P. Moulin, 2006.Low - Complexity image denoising based on statistical Modeling wavelet coefficients. IEEE Signal Trans. Image Proce,. Vol15, pp.654-665.

[16] L. Sendur and I. W. Selesnick, 2002. Bivariate shrinkage with local varience estimation. IEEE Signal Process. Lett. Vol. 9, pp. 438-441.

[17] Chen G. Y., Bui T. D., Krzyzak A. 2005. Image denoising with neighbor dependency and customized wavelet and threshold. Pattern Recognition, Vol. 38, pp.115-124.

[18] J. S. Bhat, B.N. Jagadale, Lakshminarayan H. K. 2010. Image De-noising with an Optimal threshold by Decimated Wavelet transorm. IJRTET (Nov 2010) Vol.4, No.2. 